\title{
Sentinel lymph node mapping with patent blue dye in patients with breast cancer: a retrospective single institution study
}

\author{
Fabrice Olivier ${ }^{1 \#}$, Audrey Courtois ${ }^{1 \#}$, Veronique Jossa ${ }^{2}$, Gaelle Bruck ${ }^{3}$, Samy Aouachria ${ }^{4}$, \\ Michel Coibion $^{5 *}$, Guy Jerusalem ${ }^{6 *}$ \\ ${ }^{1}$ Medical Oncology Department, University Hospital of Liege, Liege, Belgium; ${ }^{2}$ Anatomo-pathology Department, CHC Montlegia, Liege, Belgium; \\ ${ }^{3}$ Gynecology Department, CHR Citadelle, Liege, Belgium; ${ }^{4}$ Medical Direction Department, CHC Montlegia Liege, Belgium; ${ }^{5}$ Gynecology \\ Department, CHC Montlegia, Liege, Belgium; ${ }^{6}$ Medical Oncology Department, CHU Liège and Liège University, Liege, Belgium \\ Contributions: (I) Conception and design: V Jossa, M Coibion, G Jerusalem; (II) Administrative support: F Olivier, S Aouachria; (III) Provision of \\ study materials or patients: M Coibion, G Jerusalem; (IV) Collection and assembly of data: F Olivier, S Aouachria, G Bruck; (V) Data analysis and \\ interpretation: F Olivier, A Courtois, G Bruck, M Coibion, G Jerusalem; (VI) Manuscript writing: All authors; (VII) Final approval of manuscript: All \\ authors. \\ \#These authors contributed equally to this work and are co-first authors. \\ *These authors are co-last authors. \\ Correspondence to: Audrey Courtois. Medical Oncology Department, University Hospital of Liege, Avenue de l’Hopital, 1, 4000 Liege, Belgium. \\ Email: a.courtois@chuliege.be.
}

Background: Since the end of the last century, sentinel lymph node biopsy (SLNB) has replaced axillary lymph node dissection (ALND) as standard of care for axillary staging in early breast cancer in patients without any clinical sign of axillary lymph node infiltration. The worldwide most frequently used mapping method consists in the injection of radioactive technetium-99 isotope alone or in combination with blue dye. As a specific infrastructure and dedicated personnel are needed for the use of a radioactive tracer, the $\mathrm{CHC}$ in Liege (Belgium) decided to test the use of patent blue dye alone to detect sentinel lymph nodes in a large consecutive cohort of patients and compared the results with radioactive mapping methods and guidelines recommendations.

Methods: Patent blue dye was used in 456 consecutive patients with early breast cancer who underwent conservative breast cancer surgery or radical mastectomy between 1/1/2000 and 31/12/2007 in a community hospital (CHC Liège, Belgium). After SLNB, an ALND was performed in each patient.

Results: Sentinel lymph nodes were identified in 444 patients among the 456 patients evaluated by this mapping method during this time period, which represents a detection rate of $97.4 \%$. Infiltrated lymph nodes were detected in $32.7 \%$ of patients (149/456) while in the 444 patients with sentinel lymph nodes identified and resected, 137 patients have at last one positive lymph node (30.9\%). The false negative rate was $4.9 \%$ and the predictive negative value was $97.7 \%$ with the blue dye mapping method.

Conclusions: In addition of the simplicity of the method and the large economic advantage, SNLB using blue dye alone showed a quite acceptable performance in our retrospective analysis concerning its ability to find the SLN as well as its reliability to remove the good ones.

Keywords: Early breast cancer; sentinel lymph nodes biopsy (SLNB); axillary lymph nodes dissection (ALND); blue dye

Submitted Jun 22, 2021. Accepted for publication Aug 04, 2021.

doi: $10.21037 /$ gs-21-415

View this article at: https://dx.doi.org/10.21037/gs-21-415 


\section{Introduction}

Axillary lymph node infiltration represents the one of most important prognostic factors in breast cancer. In patients with early-breast cancer without clinical signs of node metastases, sentinel lymph node biopsy (SLNB) has progressively replaced axillary lymph node dissection (ALND) as the standard of care for the evaluation of axillary lymph node infiltration. Cabanas (1) introduced this new concept in 1977 and development of mapping methods since the 1990s allowed it widespread use with high sensitivity $(2,3)$. The SLNB reduced morbidity and magnitude of lymph node resection without compromising the oncological outcomes (4). Different methods of tracing were developed to map sentinel nodes with first of all the blue dye method, then a radioisotope method with technetium-99 or a combination of both $(5,6)$.

Several studies have compared SLNB and ALND but the NSABP B-32 study published in 2007 by Krag et al. is used as the reference (7). In this study, the conventional ALND was compared with SLNB. SLN were identified with the combination of blue dye and radioisotope method. The detection rate of SLN in this study was $97.2 \%$. This result is used as reference for clinical practice. Indeed, the ESMO clinical guidelines recommend that a detection rate of lymph nodes higher than $97 \%$ must be obtained whatever the mapping method used (8).

Although the most widespread mapping method consists in the use of technetium-99 alone or in combination with blue dye, it needs the presence of a nuclear medicine center close to the surgical center which is not always possible notably in developing countries. Moreover, the cost of this radio-tracing technique is largely higher than blue dye alone and the use of radioisotopes is less and less appreciated in the medical field because of the exposure to radiation, not only during medical use but also and mainly during tracer's preparation in the lab. An alternative could be the use of blue dye alone. However, only studies with a very limited number of participants reviewed in a recent meta-analysis show results using exclusively this tracer (9). Experience with any mapping method may be a critical factor in order to observe the highest detection and lowest false-negative rate. Consequently, data from larger studies are needed.

In a community hospital in Liège (Belgium), after having used technetium in the 90ties, we introduced the blue dye which we used as lonely tracer from 01/01/2000.

In the present report, we presented results from a large cohort of patients who underwent radical mastectomy or breast conservative surgery between 1/1/2000 and
$31 / 12 / 2007$. Only blue dye was used in these patients to detect SLN and a complete ALND was performed after the SNL biopsy in each patient. The detection rate, the false-negative rate and the predictive negative value were measured and compared to the results of the NSABP B-32 to validate blue dye alone as a high quality tracer in order to use it as reference methods in future studies.

We present the following article in accordance with the STROBE reporting checklist (available at https://dx.doi. org/10.21037/gs-21-415).

\section{Methods}

\section{Patients}

This retrospective study reviewed all consecutive women who underwent breast conservative surgery or mastectomy between 1/1/2000 and 31/12/2007 in a community hospital in Liège (CHC Liege, Belgium). Patients having received neoadjuvant treatment were excluded from the study. All exclusively female patients presented an invasive breast cancer without any clinical and sonographic signs of axillary lymph node invasion. All patients underwent SLN resection followed by ALND. The study was conducted in accordance with the Declaration of Helsinki (as revised in 2013). The study was approved by the ethics board of CHC Liege (n²0/42/105) and individual consent for this retrospective analysis was waived.

\section{Sentinel lymph node mapping and resection}

The surgery was performed under general anaesthesia. Detection of SLN was realized by the injection of $2 \mathrm{~mL}$ of $2.5 \%$ diluted patent blue dye under the dermis directly above the tumor and also in the parenchyma of the breast at the 4 quadrants of the peritumoral area. In the case of non-palpable tumors, the radiologist indicated the site of tumor and the injection was realized with the same process. Directly after the patent blue injection, a manual massage was then performed during 1 minute. Lymph nodes with bright and saturated blue coloration and located at level 1 of Berg were then removed and called "principal sentinel lymph nodes" (PSN) while the other nodes with detection of pale blue color located deeper in level 1 or higher at level 2 of Berg were also biopsied and called "accessory sentinel lymph nodes" (ASN, Figure 1). An ALND was then realized. Thereafter breast conservative surgery or mastectomy was realized. 


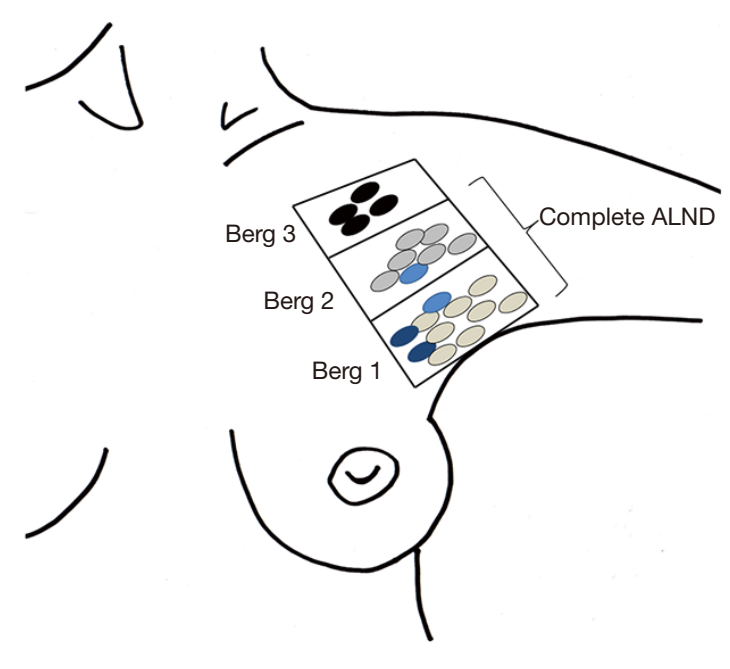

Figure 1 Principal and accessory sentinel lymph node localizations. Principal sentinel lymph nodes correspond to blue labelled nodes at the Berg 1 level (in dark blue). Accessory lymph nodes correspond to blue labelled nodes localized in the deep Berg 1 level and in the Berg 2 level (in light blue). Additional axillary lymph node dissection (ALND) corresponds to the resection of all other lymph nodes found at the Berg 1 and Berg 2 levels not labelled in blue (in grey). Lymph nodes at the Berg level 3 (in black) are not removed.

\section{Patbological analysis}

All nodes with size less than $10 \mathrm{~mm}$ were cut in two pieces while nodes with larger size were cut in three pieces. All samples were fixed in formol $4 \%$ and included in paraffin. Sections of $4 \mu \mathrm{m}$ were realized and one of them was stained with hematoxylin-eosin for the search of metastases. In the case that all resected lymph nodes from a patient were negative, a specific immunostaining was realized using the monoclonal antibody against cytokeratin KL1 (DAKO, Denmark). This technic allows the detection of micrometastasis in lymph nodes.

\section{Measures and statistical analyses}

Results were expressed as mean \pm standard deviation (SD) for each variable. The detection rate corresponded to the number of patients with at last one sentinel lymph nodes biopsied divided by the total number of patients. The number of positive lymph nodes corresponded to the number of patients in whom metastasis was detected in at last one lymph node (SLN and ALND). A false negative event is a case with positive lymph nodes in whom SLN were identified as negative. The rate of false-negative SLN biopsy was calculated as $(\mathrm{FN} / \mathrm{FN}+\mathrm{TP}) \times 100$, where $\mathrm{FN}$ is the number of false negatives and TP the number of true positives. The sensibility was measured as TP/TP + FN Finally, the negative predictive value corresponded to the proportion of negative results that were true negative $(\mathrm{TN})$ and was calculated as $(\mathrm{TN} / \mathrm{TN}+\mathrm{FN})$.

\section{Results}

A total of 456 consecutive women with breast cancer were retrospectively included in this study (Table 1). The mean age \pm standard deviation $(\mathrm{SD})$ was $55.2 \pm 11.4$ years with $67.5 \%$ of women older than 50 years. Mastectomy was performed in 159 patients $(34.9 \%)$ and breast conservative surgery in 297 patients $(65.1 \%)$. The large majority of cancer was ductal carcinoma (82\%) and the mean tumor size $\pm \mathrm{SD}$ was $1.7 \pm 1.0 \mathrm{~cm}$. The histological score of Bloom, the localization of the tumors, hormone receptor expression and HER2 status are detailed in Table 1.

The patent blue highlighted the mapping of PSN in 444 women indicating a global detection rate of SLN of $97.4 \%$ (Table 2). The mean number \pm SD of detected PSN was $1.8 \pm 1.1$. Accessory sentinel lymph nodes (ASN) were identified in 351 women (detection rate: $77.0 \%$ ) with a total mean number of nodes \pm SD by patient of $1.5 \pm 1.3$. In total, patent blue allowed the mapping of a mean number \pm SD of $3.3 \pm 1.7$ sentinel nodes (SLN) by patient.

Positive lymph nodes for metastases were found in $149 / 456$ patients (32.7\%). In the 444 patients with detected SLN, 131 have at last one positive lymph node (29.5\%) in principal sentinel lymph nodes giving a false negative rate of $9.0 \%$ and a sensitivity of $91 \%$ (Table 3). If the ASN were added, 137 patients have a positive lymph node decreasing the false negative rate to $4.9 \%$ and increasing the sensitivity to $95.1 \%$. The negative predictive value for SLN biopsy was $97.7 \%$.

The specific labelling with antibody against cytokeratin KL1 was performed in 332 patients including the 13 cases of sentinel lymph node failure. Among these 332 patients, micro-metastases were found in 42 patients with 26 patients for whom standard histology was negative for PSN, ASN and also axillary dissection (AD). The detection rate of micro-metastasis was $7.8 \%$. Among these 26 detections of micro-metastases, 24 were found in the PSN (92.3\%). The total number of patients with any metastases in lymph nodes was then 170 (37.3\%) including 161 (94.7\%) detected with the mapping of SLNs (155 in PSN and 6 in ASN). No 
Table 1 Patient and tumor characteristics

\begin{tabular}{|c|c|}
\hline Clinical characteristics & $\mathrm{N}(\%)$ \\
\hline Total & $456(100.0)$ \\
\hline Age (years), mean \pm SD & $55.2 \pm 11.4$ \\
\hline$<50$ & $148(32.5)$ \\
\hline$\geq 50$ & $308(67.5)$ \\
\hline \multicolumn{2}{|l|}{ Laterality } \\
\hline Left & $226(49.6)$ \\
\hline Right & $230(50.4)$ \\
\hline Tumor size $(\mathrm{cm})$, mean \pm SD & $1.7 \pm 1.0$ \\
\hline \multicolumn{2}{|l|}{ Tumor localization } \\
\hline Multicentric & $156(34.2)$ \\
\hline Superior (external/internal/central) & $111 / 50 / 41(24.3 / 11.0 / 9.0)$ \\
\hline Inferior (external/internal/central) & 22/23/10 (4.8/5.0/2.2) \\
\hline Midline (external/internal) & $20 / 10(4.4 / 2.2)$ \\
\hline Areolar or retro-areolar & $13(2.6)$ \\
\hline \multicolumn{2}{|l|}{ Bloom score } \\
\hline 1 & $144(31.6)$ \\
\hline 2 & $180(39.5)$ \\
\hline 3 & $93(20.4)$ \\
\hline UNK & $39(8.6)$ \\
\hline \multicolumn{2}{|l|}{ Cancer type } \\
\hline Ductal carcinoma & $374(82.0)$ \\
\hline Lobular carcinoma & $66(14.5)$ \\
\hline Others* & $16(3.5)$ \\
\hline \multicolumn{2}{|l|}{ Type of surgery } \\
\hline Mastectomy & $159(34.9)$ \\
\hline Breast conservative surgery & $297(65.1)$ \\
\hline \multicolumn{2}{|l|}{ Estrogen receptor (ER) } \\
\hline ER positive & $397(87.1)$ \\
\hline ER negative & $57(12.5)$ \\
\hline UNK & $2(0.4)$ \\
\hline \multicolumn{2}{|l|}{ Progesterone receptor (PR) } \\
\hline PR positive & $144(31.6)$ \\
\hline PR negative & $310(68.0)$ \\
\hline UNK & $2(0.4)$ \\
\hline \multicolumn{2}{|l|}{ HER 2 status } \\
\hline HER2 amplified & $66(14.5)$ \\
\hline HER2 negative & $390(85.5)$ \\
\hline
\end{tabular}

*, tubular carcinoma, canalicular-lobular carcinoma, carcinoma canalicular infiltrant, mucinous carcinoma, tubulolobular carcinoma, papillary carcinoma, colloidal carcinoma, micropapillary canalicular carcinoma, adenosquamous carcinoma. UNK, unknown; HER2, human epithelial growth factor receptor 2 . side effect such as allergic reactions or persisting blue skin color were observed in our study.

The study was conducted during 8 years and the longitudinal study did not show any difference in term of number of sentinel node detection over time while the number of nodes included in $\mathrm{AD}$ decreased over time due to increased surgeon experience and trend to treatment de-escalation in the field of surgery (mean of 11.9 nodes resected during the first year and 5.9 during the last year, data not shown). Moreover, the detection rate stayed around $97 \%$ each year while the false negative rate tended to decrease during the last 4 years (Table 4). Finally, a false negative SLN result did not seem to be associated with any clinicopathological factor (Table 5).

\section{Discussion}

Since the development of mapping methods during the nineties, the SLN biopsy has become the gold standard for the axillary lymph node staging evaluation in patients with early-breast cancer without any evidence of lymph node metastases. The SLN biopsy allows to decrease strongly the morbidity generated by ALND. The NSABP B-32 trial published in 2007 remains the reference for the definition of standard of care (7). The NSABP B-32 prospective study was conducted on a large cohort of 5611 patients presenting early-breast cancer without clinical evidence of lymph node invasion. The mapping method used was the combination of the injection of technetium-99m associated with the isoflurane blue injection. After the resection of SLN, a total ALND was performed and revealed a detection rate of $97.2 \%$ of SLN. This study became a reference for sentinel node mapping and recent guidelines recommend a detection rate of at least $97 \%$ for an appropriate mapping method (8). Two other important factors to determine the quality of the sentinel lymph node mapping are the measure of the false negative rate which corresponds to the percent of risk to miss an infiltrated lymph node and predictive negative value which represents the proportion of true negative among the negative results. In the NSABP B-32 the false negative rate was $9.8 \%$ with a predictive negative value of $96.1 \%$. Technetium-99 alone or in combination with blue dye became the worldwide predilection technic this last decade. However the green wave observed during these last years tends to privilege natural tracers compared to a radioactive isotope.

Vital blue dye has been evaluated in comparison with a combined technic with radioisotopes in relatively small 
Table 2 Detection rates of sentinel lymph nodes

\begin{tabular}{lccc}
\hline Lymph nodes types & $\mathrm{N}$ & Detection rate (\%) & Mean number of SLN \\
\hline Principal sentinel lymph nodes (PSN) & $444 / 456$ & 97.4 & $1.8 \pm 1.1$ \\
Accessory sentinel lymph nodes (ASN) & $351 / 456$ & 77 & $1.5 \pm 1.3$ \\
Total (PSN + ASN) & $444 / 456$ & 97.4 & $3.3 \pm 1.7$ \\
NSABP B-32 (7) & $5,379 / 5,536$ & 97.2 & $2.8 \pm 2.1$ \\
\hline
\end{tabular}

SLN, sentinel lymph node.

Table 3 Positive lymph nodes and false negative rate

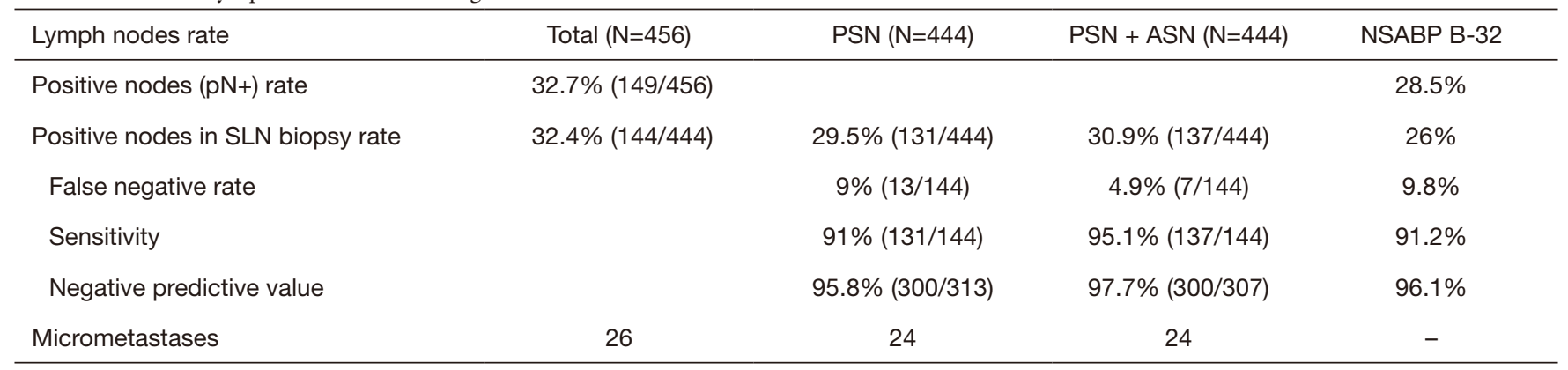

PSN, principal sentinel lymph nodes; ASN, accessory sentinel lymph nodes.

Table 4 Consistent results over time

\begin{tabular}{|c|c|c|c|c|c|c|c|}
\hline Year & Patients (N) & $P S N+A S N$ & Positive SLN & $\begin{array}{c}\mathrm{pN}+\text { total }(\mathrm{SLN}+ \\
\mathrm{AD})\end{array}$ & $\begin{array}{c}\mathrm{pN}+\text { total in } \\
\text { patient with } \mathrm{PSN}\end{array}$ & $\begin{array}{c}\text { SLN detection rate } \\
(\%)\end{array}$ & FNR (\%) \\
\hline 2000 & 38 & 38 & 6 & 8 & 8 & 100 & 25 \\
\hline 2001 & 45 & 44 & 13 & 13 & 13 & 97.8 & 0 \\
\hline 2002 & 43 & 42 & 17 & 17 & 17 & 97.7 & 0 \\
\hline 2004 & 68 & 66 & 17 & 18 & 18 & 97.1 & 5.6 \\
\hline 2005 & 60 & 58 & 20 & 22 & 21 & 97.7 & 4.8 \\
\hline 2006 & 74 & 72 & 26 & 28 & 27 & 97.3 & 3.7 \\
\hline 2007 & 68 & 66 & 20 & 21 & 21 & 97.1 & 4.8 \\
\hline
\end{tabular}

PSN, principal sentinel lymph node; ASN, accessory sentinel lymph node; $\mathrm{pN}+$, positive lymph node; SLN, sentinel lymph node; AD, axillary dissection; FNR, false negative rate.

cohorts of patients $(10,11)$. Ang et al. realized the largest study in 2014 with a cohort of 170 patients during 5 years for whom blue dye was used alone to detect SLN (12). In this study the overall detection rate was $90.6 \%$ however, this rate tended to increase each years and arrived to $95 \%$ the last year of the study. This result suggests that experience with a specific mapping method is very important in order to see the best performance.
To the best of our knowledge, the present study analyzed SLN biopsy using patent blue dye alone in the largest cohort of patients with early breast cancer ever reported. A total of 456 patients underwent surgery and SLN were identified in 444 of them. We found a detection rate of $97.4 \%$ similar to what has been reported in the NSABP B-32 study (7). The false negative rate of SLN mapping with blue dye was $4.9 \%$ which was around two times lower 
Table 5 Association between clinicopathological factors and lymph node infiltration

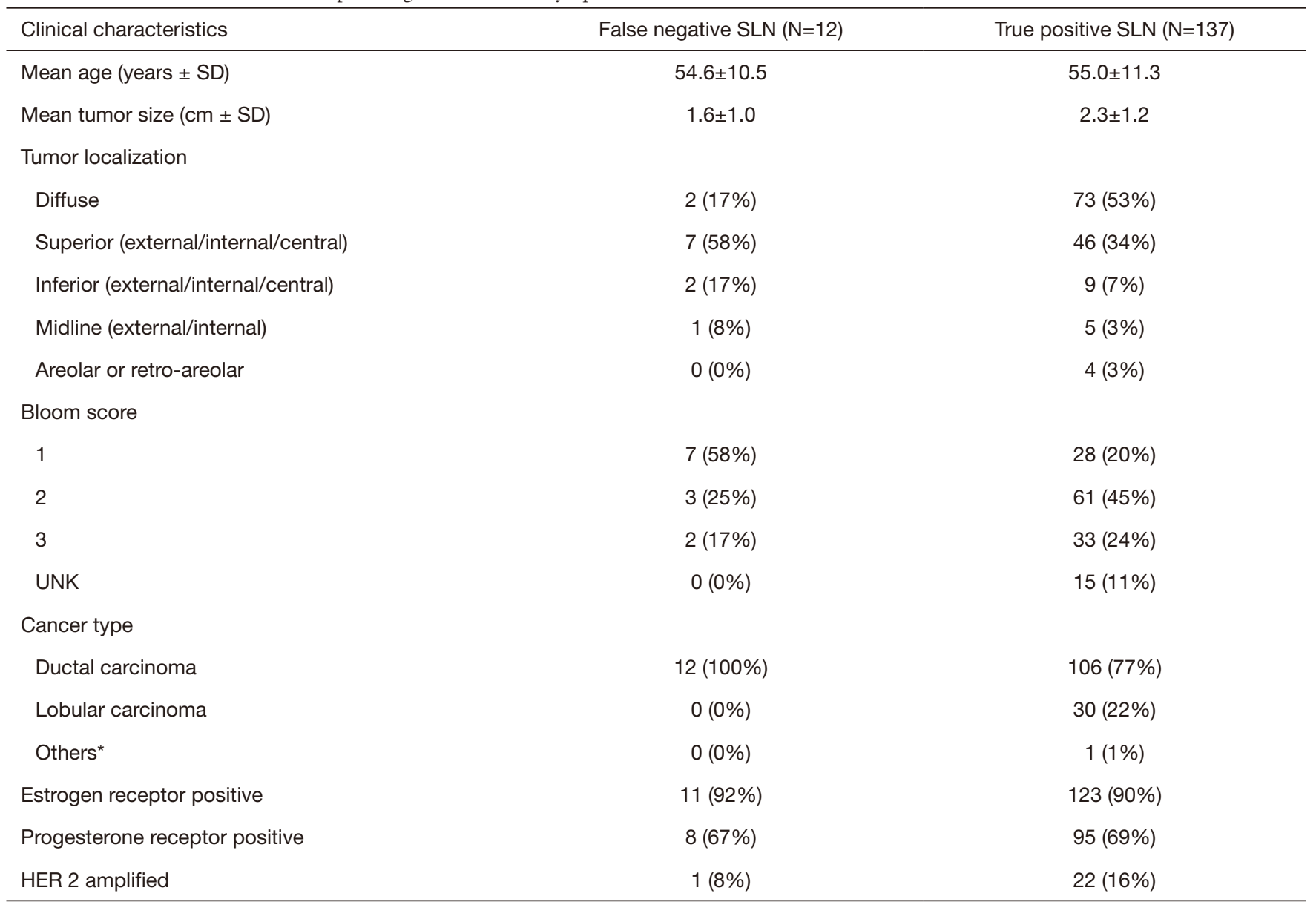

*, tubular carcinoma, canalicular-lobular carcinoma, carcinoma canalicular infiltrant, mucinous carcinoma, tubulolobular carcinoma, papillary carcinoma, colloidal carcinoma, micropapillary canalicular carcinoma, adenosquamous carcinoma. SLN, sentinel lymph node; $\mathrm{SD}$, standard deviation; UNK, unknown.

than in the reference study NSABP B-32 (9\%). Moreover the predictive negative value was $97.7 \%$ higher than in the NSABP B-32 trial (96.1\%). The false negative lymph nodes were not associated with specific clinicopathological factors.

An originality of our study is the identification and excision of two types of SLN, PSN resected at the Berg 1 level and ASN found at the Berg 1 or 2 level. Among the 444 patients with SLN identified (PSN + ASN), 137 patients had at least 1 lymph node infiltrated $(29.5 \%$, 131 patients present an infiltrated PSN and 6 patients an ASN) while among the total cohort of 456 patients who underwent an ALND, 149 patients presented at last one positive node $(32.7 \%)$. Moreover, the search for micrometastases with specific immuno-labelling allowed the detection of 26 additional patients presenting a more limited lymph node infiltration and in 24 of them (92.3\%) the micro-metastases were detected in PSN.

Data from the NSABP B-32 study demonstrated a relation between the false negative rate and the number of sentinel lymph nodes removed. In this study, this rate was $17.7 \%$ in case of 1 node, $10 \%$ with 2 nodes, $6.9 \%$ with 3 nodes, $5.5 \%$ with 4 nodes and decreased to $1 \%$ with 5 or more nodes removed. The mean number of sentinel nodes excised in this study was 2.8 with a global false negative rate of $9.8 \%$. In our study, the mean number of principal sentinel lymph nodes dissected was 1.8 per patient associated with a false negative rate of $9 \%$. So in our study using only the patent blue dye as tracer, we obtained the same false negative rate as the reference study with less nodes excised. Moreover, the addition of accessory sentinel 
nodes increased the mean number of sentinel nodes to 3.3 leading to the decrease of false negative rate to $4.9 \%$ which is around two times lower than in the NSABP B-32 study. Although no real consensus exists concerning the number of SLN to be excised, a common practice is to try to have an average number of nodes removed close to 3 (13). Indeed, Bonneau et al. showed in 2015 an association between the excision of 3 sentinel nodes and improved survival outcomes in patient with breast cancer (14). Another study realized in 2018 showed similar results with lower recurrence-free survival with one lymph node resected compared to patients with 2 or more lymph nodes removed (15).

The longitudinal analysis of our study revealed that the false negative rate remained stable during the last 4 years. This observation suggests potentially the need of an adaptive period for the surgeon to obtain a higher performance.

After the publication of the NSABP B-32 trial in 2007, guidelines recommended to perform SLNB routinely in place of ALND if the detection rate is higher or equal to $97 \%$. As our detection rate with blue dye was higher than $97 \%$ associated with a negative rate and a predictive negative value also better than in the reference study with radioisotope, we were completely convinced of the patent blue dye as efficient sentinel blue dye mapping method in our institution. However, blue dye presents also some disadvantages including a learning curve to be efficient or the risk to allergic reactions or persisting blue skin color observed after surgery. Since a few years, new promising techniques of SLN mapping have emerged including fluorescent dye (indocyanine green fluorescence), superparamagnetic iron oxide nanoparticles (SPIO) or contrastenhanced ultrasound (CEUS) imaging (16). In recent reports, these new mapping methods show similar or better performance of detection than blue dye alone or in combination with a radioisotope (16-18). The present study could be used as reference for future trials to test other new mapping methods.

\section{Conclusions}

The need to decrease the use of radioactive markers and to increase the accessibility of SLN mapping in all healthcare center including also in poor countries allowed the rediscovery of patent blue dye as tracer. In addition to the technical simplicity, the use of blue dye had a really great economic interest as its cost is around 20 times lower than a radioactive isotope using technic. Our study revealed that in experienced hand, blue dye alone performs as well as radioactive tracer or dual method of mapping without the need of special equipment and additional personnel.

\section{Acknowledgments}

Funding: None.

\section{Footnote}

Reporting Checklist: The authors have completed the STROBE reporting checklist. Available at https://dx.doi. org/10.21037/gs-21-415

Data Sharing Statement: Available at https://dx.doi. org/10.21037/gs-21-415

Conflicts of Interest: All authors have completed the ICMJE uniform disclosure form (available at https://dx.doi. org/10.21037/gs-21-415). The authors have no conflicts of interest to declare.

Ethical Statement: The authors are accountable for all aspects of the work in ensuring that questions related to the accuracy or integrity of any part of the work are appropriately investigated and resolved. The study was conducted in accordance with the Declaration of Helsinki (as revised in 2013). The study was approved by the local ethics board of CHC Liege ( $\left.{ }^{\circ} 20 / 42 / 105\right)$ and individual consent for this retrospective analysis was waived.

Open Access Statement: This is an Open Access article distributed in accordance with the Creative Commons Attribution-NonCommercial-NoDerivs 4.0 International License (CC BY-NC-ND 4.0), which permits the noncommercial replication and distribution of the article with the strict proviso that no changes or edits are made and the original work is properly cited (including links to both the formal publication through the relevant DOI and the license). See: https://creativecommons.org/licenses/by-nc-nd/4.0/.

\section{References}

1. Cabanas RM. An approach for the treatment of penile carcinoma. Cancer 1977;39:456-66.

2. Morton DL, Wen DR, Wong JH, et al. Technical details of intraoperative lymphatic mapping for early stage 
melanoma. Arch Surg 1992;127:392-9.

3. Alex JC, Krag DN. Gamma-probe guided localization of lymph nodes. Surg Oncol 1993;2:137-43.

4. Zahoor S, Haji A, Battoo A, et al. Sentinel Lymph Node Biopsy in Breast Cancer: A Clinical Review and Update. J Breast Cancer 2017;20:217-27.

5. Giuliano AE, Kirgan DM, Guenther JM, et al. Lymphatic mapping and sentinel lymphadenectomy for breast cancer. Ann Surg 1994;220:391-8; discussion 398-401.

6. Albertini JJ, Lyman GH, Cox C, et al. Lymphatic mapping and sentinel node biopsy in the patient with breast cancer. JAMA 1996;276:1818-22.

7. Krag DN, Anderson SJ, Julian TB, et al. Technical outcomes of sentinel-lymph-node resection and conventional axillary-lymph-node dissection in patients with clinically node-negative breast cancer: results from the NSABP B-32 randomised phase III trial. Lancet Oncol 2007;8:881-8.

8. Cardoso F, Kyriakides S, Ohno S, et al. Early breast cancer: ESMO Clinical Practice Guidelines for diagnosis, treatment and follow-up†. Ann Oncol 2019;30:1194-220.

9. Li J, Chen X, Qi M, et al. Sentinel lymph node biopsy mapped with methylene blue dye alone in patients with breast cancer: A systematic review and meta-analysis. PLoS One 2018;13:e0204364.

10. Syme DB, Collins JP, Mann GB. Comparison of blue dye and isotope with blue dye alone in breast sentinel node biopsy. ANZ J Surg 2005;75:817-21.

11. Radovanovic Z, Golubovic A, Plzak A, et al. Blue dye versus combined blue dye-radioactive tracer technique in

Cite this article as: Olivier F, Courtois A, Jossa V, Bruck G, Aouachria S, Coibion M, Jerusalem G. Sentinel lymph node mapping with patent blue dye in patients with breast cancer: a retrospective single institution study. Gland Surg 2021;10(9):2600-2607. doi: 10.21037/gs-21-415 detection of sentinel lymph node in breast cancer. Eur J Surg Oncol 2004;30:913-7.

12. Ang CH, Tan MY, Teo C, et al. Blue dye is sufficient for sentinel lymph node biopsy in breast cancer. Br J Surg 2014;101:383-9; discussion 389.

13. Dixon JM, Cartlidge CWJ. Twenty-five years of change in the management of the axilla in breast cancer. Breast J 2020;26:22-6.

14. Bonneau C, Bendifallah S, Reyal F, et al. Association of the number of sentinel lymph nodes harvested with survival in breast cancer. Eur J Surg Oncol 2015;41:52-8.

15. Kim MK, Park HS, Kim JY, et al. The clinical implication of the number of lymph nodes harvested during sentinel lymph node biopsy and its effects on survival outcome in patients with node-negative breast cancer. Am J Surg 2017;214:726-32.

16. Mok CW, Tan SM, Zheng Q, et al. Network meta-analysis of novel and conventional sentinel lymph node biopsy techniques in breast cancer. BJS Open 2019;3:445-52.

17. Guo J, Yang H, Wang S, et al. Comparison of sentinel lymph node biopsy guided by indocyanine green, blue dye, and their combination in breast cancer patients: a prospective cohort study. World J Surg Oncol 2017;15:196.

18. Zhou Y, Li Y, Mao F, et al. Preliminary study of contrastenhanced ultrasound in combination with blue dye vs. indocyanine green fluorescence, in combination with blue dye for sentinel lymph node biopsy in breast cancer. BMC Cancer 2019;19:939. 